\title{
EXTRAÇÃO DO SUMO DA BETA VULGARIS COMO ALTERNATIVA SUSTENTÁVEL NA PRODUÇÃO DE TINTAS
}

\author{
Gabriela Camila Santana Ribeiro de Lima ${ }^{1}$ \\ Ingrid Januário Santana ${ }^{2}$ \\ Leticia Gabriele Ereno ${ }^{3}$ \\ Maria Laura Castro Villa ${ }^{4}$ \\ Neiva Dias Ambrósio 5 \\ Jobair Rangel ${ }^{6}$
}

Resumo: O trabalho apresenta a extração do sumo da beterraba a partir de um método simples ao mais complexo, sendo utilizado apenas a pigmentação arrocheada para a fabricação de tinta natural, sem corantes para que não deteriore o material. Teremos a infusão da beterraba, deixando que aconteça naturalmente por minutos, dias ou semanas, para que possamos obter um pigmento completamente natural, utilizando também um aparelho, cujo nome é soxhlet, tendo a propriedade de extrair o sumo de lipídios em apenas algumas horas, dependendo do tamanho que seja o lipídio utilizado. Após a pigmentação fez necessário a adição de um aglutinante com objetivo de formar uma liga. Tento como resultado uma tinta sem corantes e não prejudicial ao meio ambiente.

Palavras-chave: Beterraba; Sumo; Aglutinante; Tinta.

\footnotetext{
1 Colégios Univap - Unidade Centro Colégio Técnico "Antônio Teixeira Fernandes.”/Univap, Brasil. E-mail: gabrielasantana.ribeiro@outlook.com.

2 Colégios Univap - Unidade Centro Colégio Técnico "Antônio Teixeira Fernandes."/Univap, Brasil. E-mail: ingrids.januario@yahoo.com.

3 Colégios Univap - Unidade Centro Colégio Técnico "Antônio Teixeira Fernandes."/Univap, Brasil. E-mail: ereno_le@hotmail.com.

4 Colégios Univap - Unidade Centro Colégio Técnico "Antônio Teixeira Fernandes."/Univap, Brasil. E-mail: mariavilla248@yahoo.com.

5 Colégios Univap - Unidade Centro Colégio Técnico "Antônio Teixeira Fernandes."/Univap, Brasil. E-mail: ambrosioneiva@gmail.com.

6 Colégios Univap - Unidade Centro Colégio Técnico "Antônio Teixeira Fernandes."/Univap, Brasil. E-mail: jobairrangel@terra.com.br.
} 